\title{
Design of a Broadband Coupled-Fed Printed Dipole Antenna as an Array Element for Direction Finding Systems
}

\author{
Sungsik Wang ${ }^{1} \cdot$ Sungjun Yoo ${ }^{1, *} \cdot$ Junsik Park $^{2} \cdot$ Hongsuk Shim $^{2} \cdot$ Hosung Choo $^{1}$
}

\begin{abstract}
This article proposes the design of a coupled-fed printed dipole antenna for direction finding (DF) systems. The antenna consists of a radiating $50^{\circ}$ angled expanded-arm printed dipole as well as a fan-shaped feeder that is located on the opposite side of the radiating dipole. The $50^{\circ}$ angled expanded-arm structure results in a wide beam-width and broadband-matching characteristics. The more the arm angle is bent, the wider the beam-width pattern. Note that the expanded structure to the side of the dipole arm allows the wide beam-width of the radiation pattern. The printed dipole is then electromagnetically coupled with the fan-shaped feeder to obtain broadband-matching characteristics. To obtain additional broadband-matching characteristics, the circular cavity and the four-step tapered structures of the radiating printed dipole are carefully tuned by considering the coupled fields induced by the indirect feeding structure. The reflection coefficients are $-14.6 \mathrm{~dB}$ and $-11.9 \mathrm{~dB}$ at $1.575 \mathrm{GHz}$ and $2.4 \mathrm{GHz}$, and the boresight gains are $4.8 \mathrm{dBi}$ and $1 \mathrm{dBi}$, respectively. To confirm the DF capability of the proposed antenna, the pattern of the $4 \times 4$ arrays is observed by varying the steering angle.
\end{abstract}

Key Words: Array, Broadband Antenna, Coupled Feed, Printed Dipole, Wide Beam Pattern Antenna.

\section{INTRODUCTION}

Direction finding (DF) systems have been widely used in military applications to detect the location of enemy radio-frequency communications. Recently, they have also been used in a variety of non-military applications, such as detecting unauthorized transmitters and intentional jamming signals [1-3]. DF performances are determined in part by direction-finding algorithms that estimate the arrival direction by using the phase differences in each antenna. In addition, antenna characteristics of the array, such as the array configurations, radiation patterns, mutual coupling, and matching bandwidth, also have a significant effect on DF performance. To improve these array element properties in
DF systems, several types of antennas have been reported, including Vivaldi antennas [4], broadband patch antennas [5], dipole type antennas [6], and slotted patch antennas [7]. However, most previous studies have focused on improving the gain, high isolation, and matching characteristics, whereas the method of improving radiation patterns requiring a wide beam-width for DF systems has not yet been fully studied [8].

In this paper, we propose a design for a coupled-fed printed dipole antenna with a wide beam-width for enhancing the DF performance. The proposed antenna consists of a radiating printed dipole and a fan-shaped feeder, located on the opposite side of the radiating dipole. The expanded structure to the side of the dipole arms allows the wide beam-width of the radiation pattern [9]. The larger the arm angle is the wider the beam-

\footnotetext{
Manuscript received May 30, 2019 ; Revised July 18, 2019 ; Accepted August 8, 2019. (ID No. 20190530-043J)

${ }^{1}$ School of Electronic and Electrical Engineering, Hongik University, Seoul, Korea.

${ }^{2}$ Hanwha Systems Co. Ltd., Seongnam, Korea.

"Corresponding Author: Sungjun Yoo (e-mail: ryoonet@naver.com)
}

This is an Open-Access article distributed under the terms of the Creative Commons Attribution Non-Commercial License (http://creativecommons.org/licenses/by-nc/4.0) which permits unrestricted non-commercial use, distribution, and reproduction in any medium, provided the original work is properly cited.

(c) Copyright The Korean Institute of Electromagnetic Engineering and Science. 
width of the pattern; a $50^{\circ}$ angled expanded-arm structure results in a wide beam-width and broadband-matching characteristics. The printed dipole is then electromagnetically coupled with the feeder to obtain broadband-matching characteristics [10]. To further improve the matching characteristic, the four-step tapered structure of the printed dipole is carefully adjusted to take into account the currents and fields induced by the indirect feeding structure [10]. To demonstrate characteristics such as reflection coefficient, bore-sight gain, and radiation patterns, the proposed antenna is measured in a full anechoic chamber. Finally, we observe the beam steering property by extending the proposed antenna to a $4 \times 4$ array in order to verify its DF capability. The results confirm that the proposed antenna is suitable for the individual elements of a DF array antenna with wide beam-scanning characteristics.

\section{Proposed ANTENNA DESIGN AND MEASUREMENT}

Fig. 1 shows the geometry of the individual elements of the proposed array antenna; these include a printed dipole on an FR4 substrate $\left(\varepsilon_{r}=4.5, \tan \delta=0.018\right)$ and a fan-shaped feeder that is located on the opposite side of the dipole. To obtain the wide beam-width radiation pattern, the dipole arm is designed to be bent and expanded at angle $w_{\phi}$ in the side direction. As the angle $w_{\phi}$ increases, the beam-width also increases. In addition, matching at $1.575 \mathrm{GHz}$ and $2.4 \mathrm{GHz}$ can be improved with an additional capacitance adjustment between the arms and the ground. For broadband impedance matching, the proposed antenna employs an indirect fan-shaped feeder connected by a microstrip line, a circular cavity, and a tapered transmission line to the antenna arms. The length and thickness of the feeding line are designed considering the resonance frequency of the antenna. The design parameters are listed in Table 1.

Fig. 2 presents a comparison of the measured and simulated reflection coefficients, represented by solid and dashed lines, respectively. The reflection coefficients for each frequency are obtained from full-wave electromagnetic simulations (FEKO; Altair, Troy, MI, USA). The measured reflection coefficient has a bandwidth of $1.3 \mathrm{GHz}\left(1.36-2.49 \mathrm{GHz},\left|S_{11}\right|<-10 \mathrm{~dB}\right)$ with a fractional bandwidth of $59 \%$, and an average reflection coefficient within the bandwidth of $-14.5 \mathrm{~dB}$. The simulated reflection coefficient has a $58 \%$ fractional bandwidth $\left(1.36-2.46 \mathrm{GHz},\left|S_{11}\right|\right.$ $<-10 \mathrm{~dB}$ ) with an average of $-13.3 \mathrm{~dB}$ within the bandwidth, which is in good agreement with the measured data.

Fig. 3 represents a comparison of the measured ('+' marker) and simulated (solid line) bore-sight gains. The realized gains for each frequency are obtained from full-wave electromagnetic simulations [11]. The measured bore-sight gains have $4.8 \mathrm{dBi}$ at $1.575 \mathrm{GHz}$ and $1 \mathrm{dBi}$ at $2.4 \mathrm{GHz}$, and the gains are greater than $0 \mathrm{dBi}$ over the entire range from $1.2 \mathrm{GHz}$ to $3 \mathrm{GHz}$. The
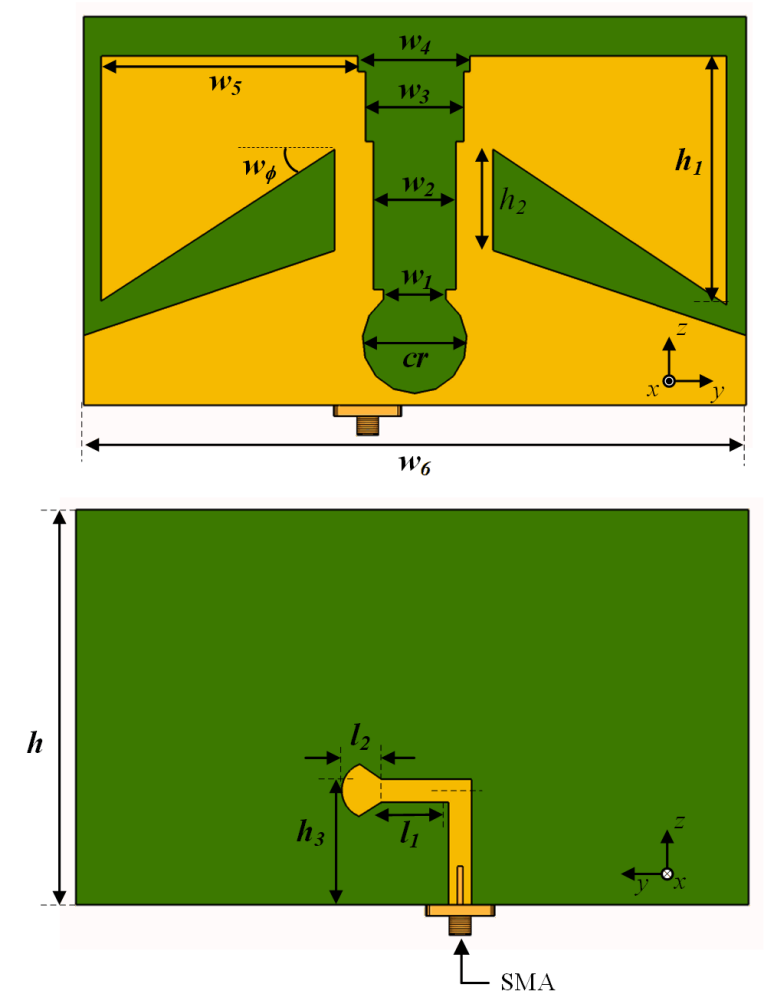

(a)

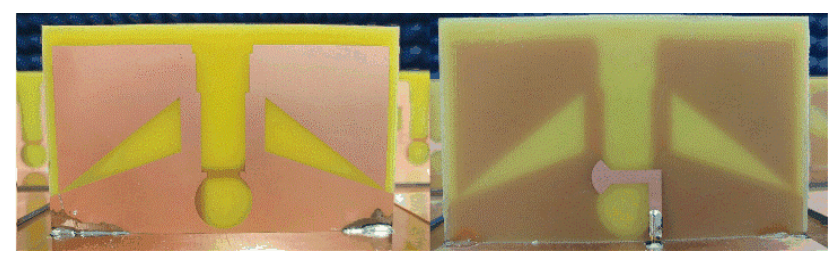

(b)

Fig. 1. The proposed printed dipole antenna's (a) top and bottom view geometry and (b) fabricated picture.

Table 1. Geometry parameters of the proposed antenna

\begin{tabular}{cc|cc}
\hline Parameter & Size $(\mathrm{mm})$ & Parameter & Size $(\mathrm{mm})$ \\
\hline$w_{1}$ & 8 & $c r$ & 14 \\
$w_{2}$ & 10.6 & $h$ & 50 \\
$w_{3}$ & 12.6 & $h_{1}$ & 34.98 \\
$w_{4}$ & 14.2 & $h_{2}$ & 13 \\
$w_{5}$ & 33 & $h_{3}$ & 15.9 \\
$w_{6}$ & 85.2 & $l_{1}$ & 2.26 \\
$w_{\phi}$ & $50^{\circ}$ & $l_{2}$ & 4.5 \\
\hline
\end{tabular}

results demonstrate that the proposed antenna can receive signals over a broad bandwidth, including applications such as mobile communications, GPS, GLONASS, and Bluetooth.

Fig. 4 shows the beam-width of the proposed antenna according to the expanded arm angle $w_{\phi}$. As the expanded arm angle $w_{\phi}$ increases, the surface current density at the side edge of the antenna arm increases. The increased surface current density at the side edges has the effect of increasing the gain in the lat- 


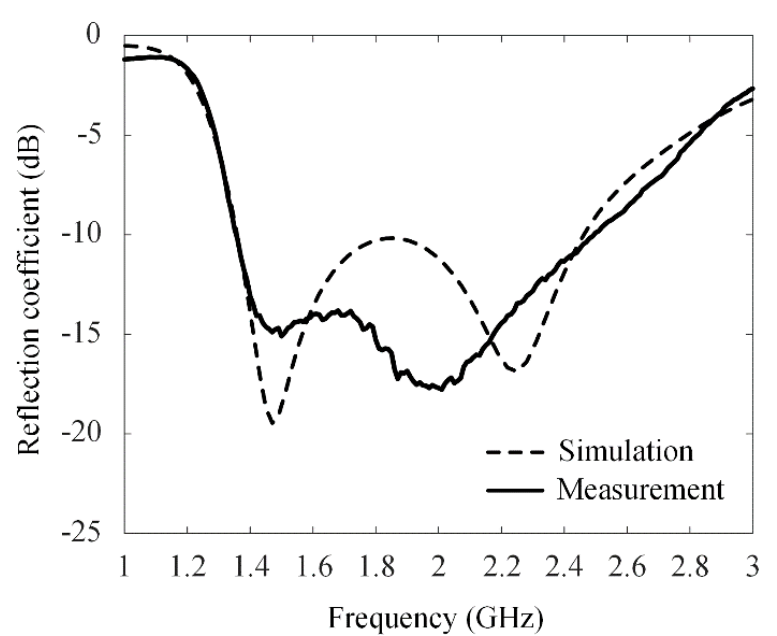

Fig. 2. Reflection coefficient of the proposed antenna.

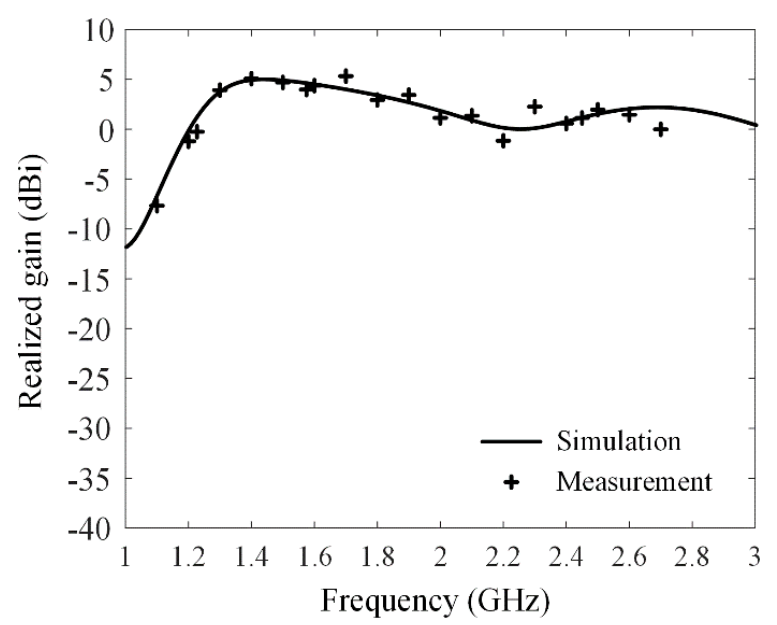

Fig. 3. Realized gain of the proposed antenna.

eral direction, resulting in a wide beam-width characteristic.

Fig. 5 shows the 2D radiation patterns of the proposed antenna in the $z x$ - and $z y$-planes. At $1.575 \mathrm{GHz}$, the measured half power beam-widths (HPBWs) are $111^{\circ}\left(z x\right.$-plane) and $112.6^{\circ}$ (zy-plane), which shows good agreement with the simulated HPBWs of $110^{\circ}$ ( $z x$-plane) and $112.5^{\circ}$ ( $z y$-plane). At $2.4 \mathrm{GHz}$, the measured patterns show slightly wider HPBWs of $174^{\circ}$ and $204^{\circ}$ on the $z x$ - and $z y$ - planes, respectively. As can be seen, the proposed antenna has a wide beam-width without significant pattern distortions in the upper hemisphere, which enables beam scanning at a wide range. To verify the beam-widening characteristics of the proposed antenna, the angle extension of the antenna arm is observed by varying the design parameters, such as the angle $w_{\phi}$. The angle extension value of the antenna wing increases as the $w_{\phi}$ is changed from $0^{\circ}$ to $50^{\circ}$, while the beamwidth dramatically increases from $78.4^{\circ}$ to $214.3^{\circ}$ at $2.4 \mathrm{GHz}$ (zy-plane).

To verify the beam scanning characteristic of the proposed

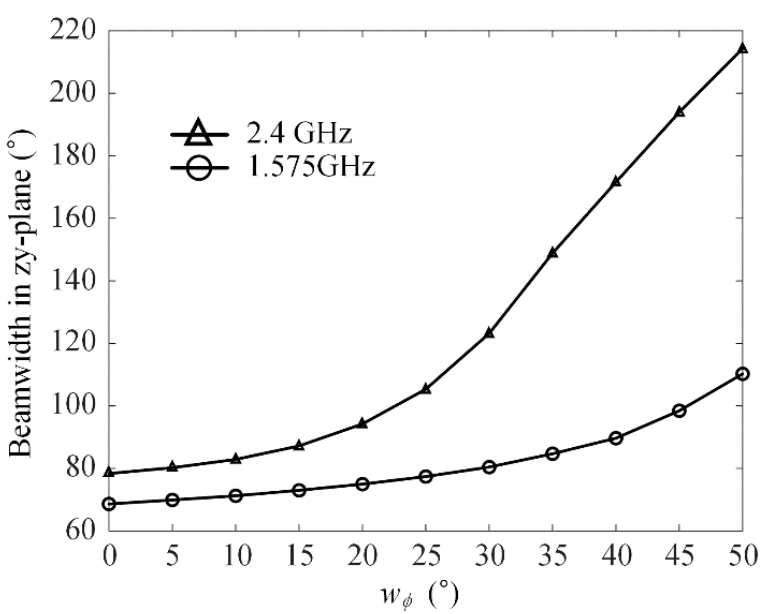

Fig. 4. Beam-width of the proposed antenna in the zy-plane according to the expanded arm angle.

antenna, the array performance is examined by extending the developed individual antennas to a $4 \times 4$ array antenna. In the array configuration, identical individual elements are spaced apart from adjacent elements by a distance of $0.47 \lambda(90 \mathrm{~mm})$.

Fig. 6 presents the beam steering patterns in the UV domain $[11,12]$, when the phase information is applied to each element of array, and the results show that the radiation pattern of the proposed array can be steered to $\theta=0^{\circ}, \phi=0^{\circ}$ and $\theta=30^{\circ}, \phi=$ $30^{\circ}$ at $1.575 \mathrm{GHz}$. The scan directions are specified by $\mathrm{U}_{0}=$ $\sin \theta_{0} \sin \phi_{0}$ and $\mathrm{V}_{0}=\sin \theta_{0} \cos \phi_{0}\left(\mathrm{U}_{0}=0\right.$ and $\mathrm{V}_{0}=0, \mathrm{U}_{0}=0.25$ and $\left.\mathrm{V}_{0}=0.43\right)$. The peak gains are $16.2 \mathrm{dBi}$ and $15.5 \mathrm{dBi}$, and the blue circle represents the maximum point of the pattern, and the yellow cross represents the target point. The side lobe levels are $3.5 \mathrm{dBi}\left(\mathrm{U}_{0}=0.77, \mathrm{~V}_{0}=0, \theta_{0}=50^{\circ}\right.$, and $\left.\phi_{0}=90^{\circ}\right)$ and $4.6 \mathrm{dBi}$ $\left(\mathrm{U}_{0}=-0.32, \mathrm{~V}_{0}=0.27, \theta_{0}=-50^{\circ}\right.$, and $\left.\phi_{0}=65^{\circ}\right)$, and the HPBWs are $27.2^{\circ}$ and $31.15^{\circ}$, respectively.

\section{CONCLUSION}

We have investigated the design of a broadband printed dipole antenna with wide beam-width characteristics for a wide beam scan angle in DF systems. The proposed antenna is composed of a radiating printed dipole and a feeder. The expanded structure to the side of the dipole arms allows it to have the wide beamwidth of the radiation pattern, and the dipole was electromagnetically coupled with the feeder for the broadband operation. The reflection coefficients are $-14.6 \mathrm{~dB}$ and $-11.9 \mathrm{~dB}$ at 1.575 $\mathrm{GHz}$ and $2.4 \mathrm{GHz}$, and the boresight gains are $4.8 \mathrm{dBi}$ and 1 $\mathrm{dBi}$, respectively. To confirm the DF capability of the proposed antenna, the pattern of the $4 \times 4$ array is observed by varying the steering angle at $\theta=0^{\circ}, \phi=0^{\circ}$ and $\theta=30^{\circ}, \phi=30^{\circ}$. The results demonstrated that the proposed antenna is suitable for the single element array in DF systems. 


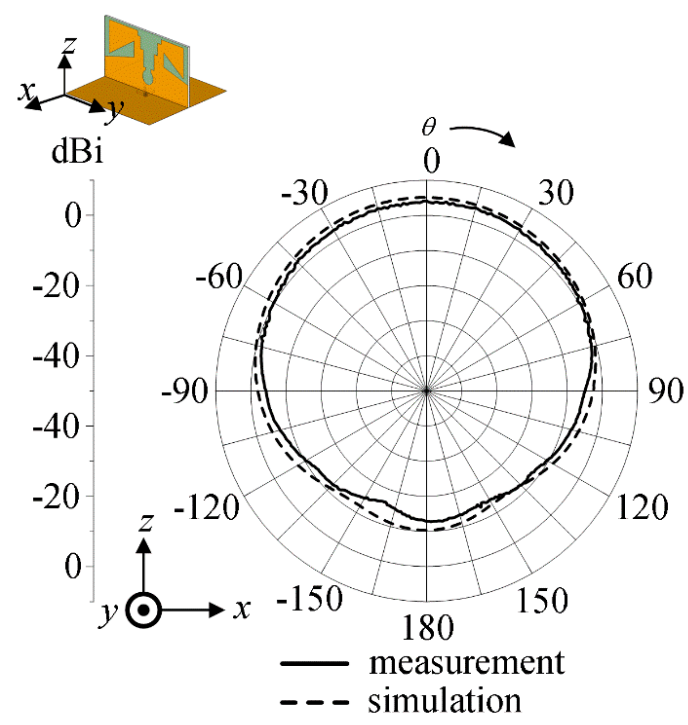

(a)

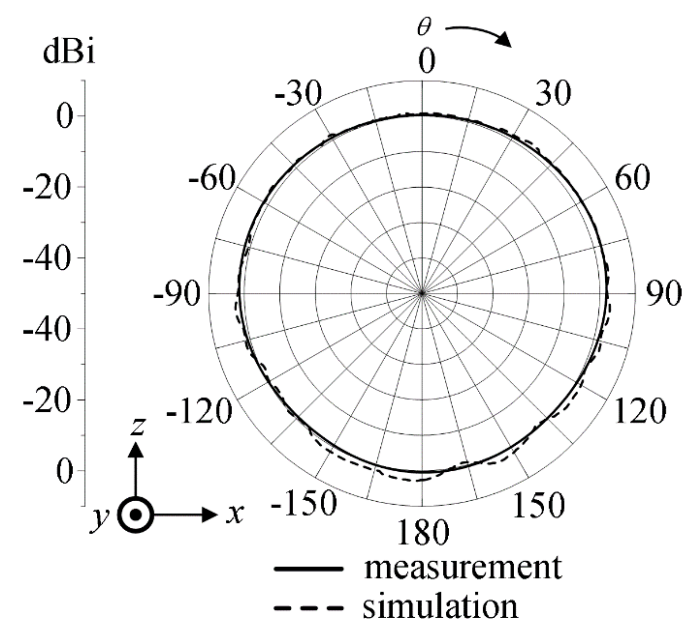

(c)

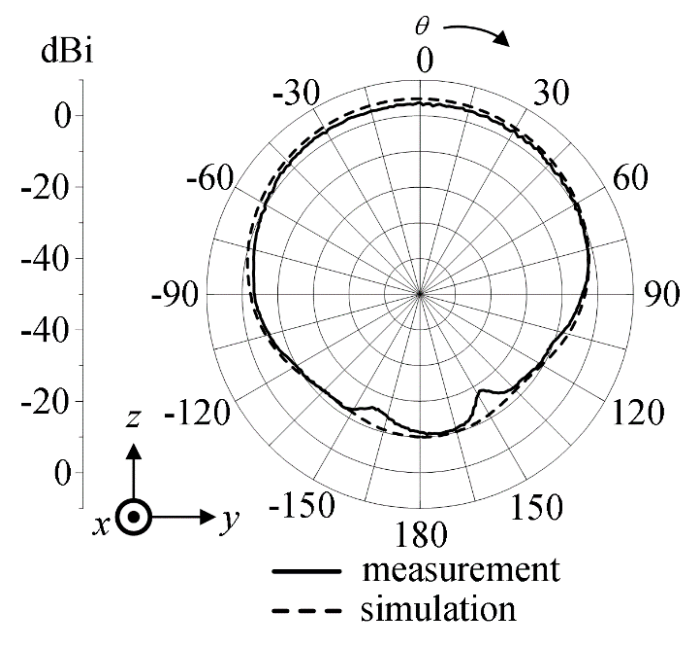

(b)

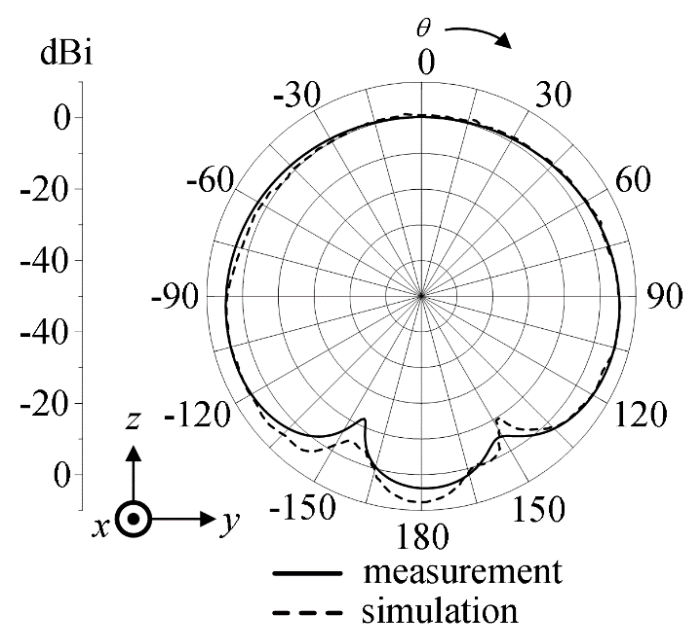

(d)

Fig. 5. Measurement and simulation of the radiation patterns in the (a) zx-plane at $1.575 \mathrm{GHz}$, (b) zy-plane at $1.575 \mathrm{GHz}$, (c) zx-plane at $2.4 \mathrm{GHz}$, and (d) zy-plane at $2.4 \mathrm{GHz}$.

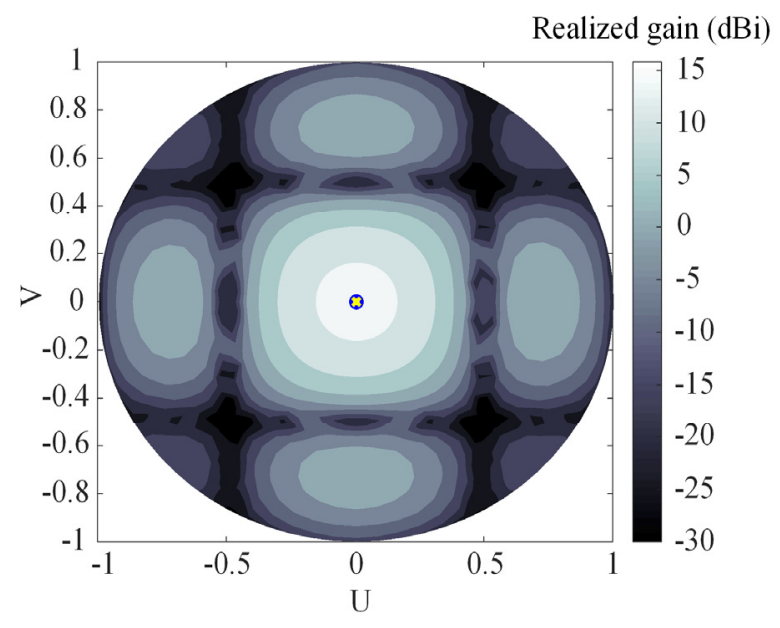

(a)

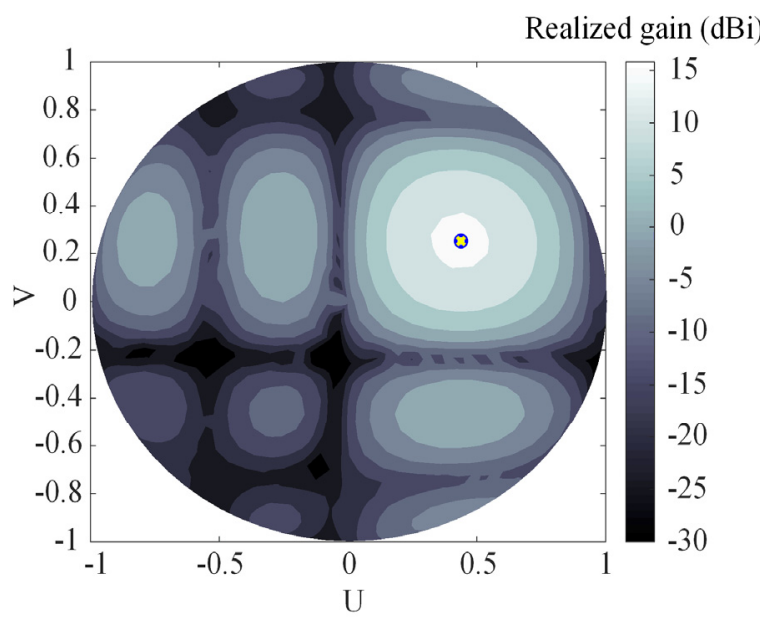

(b)

Fig. 6. Radiation pattern of the $4 \times 4$ array at $1.575 \mathrm{GHz}$ for (a) $\theta=0^{\circ}, \phi=0^{\circ}$ and (b) $\theta=30^{\circ}, \phi=30^{\circ}$. 
This work was supported by a grant-in-aid of Hanwha Systems.

\section{REFERENCES}

[1] O. Somekh, O. Simeone, Y. Bar-Ness, and W. Su, "Detecting the number of transmit antennas with unauthorized or cognitive receivers in MIMO systems," in Proceedings of IEEE Military Communications Conference, Orlando, FL, 2007, pp. 1-5.

[2] R. L. Fante and J. J. Vaccaro, "Wideband cancellation of interference in a GPS receive array," IEEE Transactions on Aerospace and Electronic Systems, vol. 36, no. 2, pp. 549-564, 2000.

[3] S. Yoo, H. Kim, G. Byun, and H. Choo, "Estimation of detection performance for vehicle FMCW radars using EM simulations," Journal of Electromagnetic Engineering and Science, vol. 19, no. 1, pp. 13-19, 2019.

[4] G. Byun, T. H. Lim, M. C. Kang, and H. Choo, "Antipodal Vivaldi antennas with foldable hinged plates for adaptive polarization and gain adjustments," Microwave and Optical Technology Letters, vol. 60, no. 1, pp. 183-187, 2018.

[5] J. Kim and Y. Sung, "Dual-band microstrip patch antenna with switchable orthogonal linear polarizations," Journal of Electromagnetic Engineering and Science, vol. 18, no. 4, pp. 215-220, 2018.

[6] J. Tao, Q. Feng, and T. Liu, "Dual-wideband magnetoelec-

\section{Sungsik Wang}

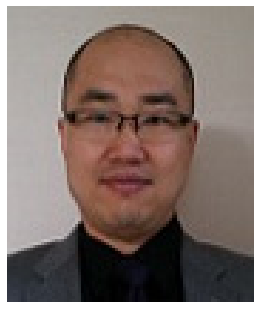

received his B.S. and M.S. degrees in radio science and engineering from Hanyang University, Seoul, Korea, in 1997 and 1999, respectively. He is currently working toward his Ph.D. in electronics and computer engineering at Hongik University, Seoul, Korea. His research interests include the beam propagation under the abnormal atmospheric phenomenon, broadband antenna design, use of the optimization algorithm in developing antennas, and antenna arrays beamforming.

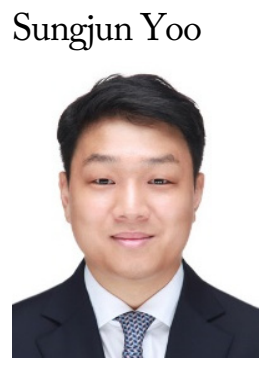

received his B.S., M.S., and Ph.D. degrees in electronic and electrical engineering from Hongik University, Seoul, Korea, in 2014, 2016, and 2019, respectively. In September 2019, he joined the Metamaterial Electronic Device Research Center, Hongik University, Seoul, Korea, where he is currently a research professor. His research interests include global positioning system antennas, antenna arrays, and position optimization of array elements for adaptive beamforming. tric dipole antenna with director loaded," IEEE Antennas and Wireless Propagation Letters, vol. 17, no. 10, pp. 1885-1889, 2018.

[7] C. R. Medeiros, E. B. Lima, J. R. Costa, and C. A. Fernandes, "Wideband slot antenna for WLAN access points," IEEE Antennas and Wireless Propagation Letters, vol. 9, pp. 79-82, 2010.

[8] R. Gardelli, G. La Cono, and M. Albani, "A low-cost suspended patch antenna for WLAN access points and pointto-point links," IEEE Antennas and Wireless Propagation Letters, vol. 3, pp. 90-93, 2004.

[9] R. A. Alhalabi and G. M. Rebeiz, "High-efficiency angleddipole antennas for millimeter-wave phased array applications," IEEE Transactions on Antennas and Propagation, vol. 56, no. 10, pp. 3136-3142, 2008.

[10] J. Shin and D. H. Schaubert, "A parameter study of stripline-fed Vivaldi notch-antenna arrays," IEEE Transactions on Antennas and Propagation, vol. 47, no. 5, pp. 879886, 1999.

[11] A. Alexopoulos and A Shaw, "Optimum configurations for static and rotating phased arrays," IEEE Transactions on Aerospace and Electronic Systems, vol. 44, no. 4, pp. 15511559, 2008.

[12] A. Alexopoulos, "Radar systems considerations for phased array aperture design using conformal transformations on Riemannian manifolds," IEEE Transactions on Antennas and Propagation, vol. 55, no. 8, pp. 2239-2246, 2007.

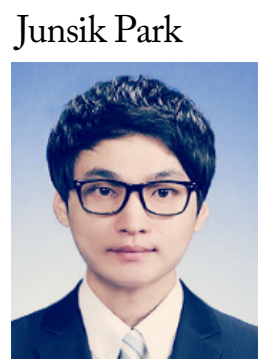
received his B.S. and M.S. degrees in electronics engineering from Chonbuk National University, Jeonju, Korea, in 2014 and 2016, respectively. He is currently working as a researcher in Hanwha Systems, Pangyo, Korea. His research interests include the electric-warfare system, electric support, electric attack, and military antenna and RF circuit.

Hongsuk Shim

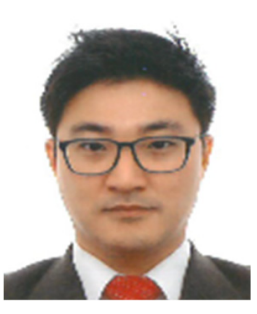

received his B.S. in computer engineering from Chungbuk National University, Cheongju, Korea in 2005 and his M.S. degree in computer engineering from Chungnam National University, Daejeon, Korea in 2008. He is currently working as a researcher in Hanwha Systems, Pangyo, Korea. His research interests include the electric-warfare system, electric support, electric attack, and ELINT/COMINT signal analysis and signal processing. 


\section{Hosung Choo}

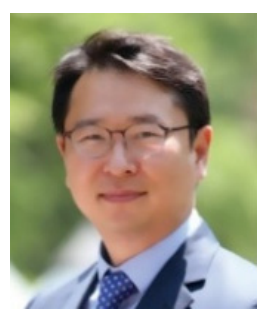

received his B.S. degree in radio science and engineering from Hanyang University in Seoul, in 1998, and his M.S. and Ph.D. degrees in electrical and computer engineering from the University of Texas at Austin, in 2000 and 2003, respectively. In September 2003, he joined the School of Electronic and Electrical Engineering, Hongik University, Seoul, Korea, where he is currently a full-time professor. His principal areas of research are the use of the optimization algorithm in developing antennas and microwave absorbers. His studies include the design of small antennas for wireless communications, reader and tag antennas for RFID, and onglass and conformal antennas for vehicles and aircraft. 